\title{
MAGIQ at the W. M. Keck Observatory: initial deployment of a new acquisition, guiding, and image quality monitoring system
}

Sean M. Adkins, Judith G. Cohen, Joel Aycock, James Bell, Richard Cohen, et al.

Sean M. Adkins, Judith G. Cohen, Joel Aycock, James Bell, Richard Cohen, Andrew Cooper, Bob Goodrich, Jimmy Johnson, Shui H. Kwok, Jim Lyke, Kevin McCann, Chris Neyman, Tom Nordin, Sergey Panteleev, Grant Tolleth, Myrna Tsubota, "MAGIQ at the W. M. Keck Observatory: initial deployment of a new acquisition, guiding, and image quality monitoring system," Proc. SPIE 7014, Ground-based and Airborne Instrumentation for Astronomy II, 70141U (9 July 2008); doi: 10.1117/12.789857

Event: SPIE Astronomical Telescopes + Instrumentation, 2008, Marseille, France 


\title{
MAGIQ at the W. M. Keck Observatory, initial deployment of a new acquisition, guiding and image quality monitoring system
}

\author{
Sean M. Adkins ${ }^{* a}$, Judith G. Cohen ${ }^{\mathrm{b}}$, Joel Aycock ${ }^{\mathrm{a}}$, James Bell ${ }^{\mathrm{a}}$, Richard Cohen ${ }^{\mathrm{a}}$, Andrew Cooper ${ }^{\mathrm{a}}$, \\ Bob Goodrich ${ }^{\mathrm{a}}$, Jimmy Johnson ${ }^{\mathrm{a}}$, Shui H. Kwok ${ }^{\mathrm{a}}$, Jim Lyke ${ }^{\mathrm{a}}$, Kevin McCann ${ }^{\mathrm{a}}$, Chris Neyman ${ }^{\mathrm{a}}$, Tom \\ Nordin $^{\mathrm{a}}$, Sergey Panteleev ${ }^{\mathrm{a}}$, Grant Tolleth ${ }^{\mathrm{a}}$, Myrna Tsubota ${ }^{\mathrm{a}}$ \\ ${ }^{\mathrm{a}}$ W. M. Keck Observatory, 65-1120 Mamalahoa Highway, Kamuela, HI, USA 96743; \\ ${ }^{\mathrm{b}}$ California Institute of Technology, M/C 105-24, Pasadena, CA 91125, USA
}

\begin{abstract}
The W. M. Keck Observatory has completed the development and initial deployment of MAGIQ, the Multi-function Acquisition, Guiding and Image Quality monitoring system. MAGIQ is an integrated system for acquisition, guiding and image quality measurement for the Keck telescopes. This system replaces the acquisition and guiding hardware and software for existing instruments at the Observatory and is now the standard for visible wavelength band acquisition cameras for future instrumentation. In this paper we report on the final design and implementation of this new system, which includes three major components: a visible wavelength band acquisition camera, image quality measurement capability, and software for acquisition, guiding and image quality monitoring. The overall performance is described, as well as the details of our approach to integrating low order wavefront sensing capability in order to provide closed loop control of telescope focus.
\end{abstract}

Keywords: Acquisition, Guiding, Wavefront Sensing

\section{INTRODUCTION}

The W. M. Keck Observatory has completed the development and initial deployment of MAGIQ, the Multi-function Acquisition, Guiding and Image Quality monitoring system. MAGIQ is an integrated system for acquisition, guiding and image quality measurement for the Keck telescopes. This system replaces the acquisition and guiding hardware and software for existing instruments at the Observatory and is now the standard for visible wavelength band acquisition cameras for future instrumentation.

The basic concepts of the MAGIQ project were first described in a WMKO proposal submitted in 2004 to NASA under the Astronomy and Physics Research and Analysis Program to replace the existing guider system at the Observatory. The proposal was successful and funding was awarded in February 2005. Replacement of the guider system was identified by the Observatory as a priority due to the various impacts that the existing system had on observing efficiency. These impacts included problems with reliability and maintainability of the existing cameras and software, the time taken from science observations for telescope focusing, and overheads due to the small field of view (FOV) provided by the cameras on the Keck I instruments.

In developing the concept for the new acquisition and guiding system we realized that there was an opportunity to benefit science operations by providing image quality monitoring features that would include the ability to determine low order image aberrations, such as focus and coma, in a continuous, near real-time fashion when not using adaptive optics. These measurements would be performed in parallel to science observations and allow implementation of continuous correction of telescope focus, a capability that we uniquely lack in comparison to all other 8 to $10 \mathrm{~m}$ class telescopes. For large telescopes constructed since the Keck telescopes the approach has been to provide one or more "facility" acquisition and guide cameras, along with capabilities ${ }^{1,2,3,4}$ to measure wavefront quality and control telescope focus during science observations. On the Keck telescopes each of the seeing limited instruments is equipped with at least one acquisition and guide camera that is integrated with the instrument. This approach facilitates the implementation of slit viewing cameras for spectrographs and reduces problems with flexure between the acquisition camera focal plane and the instrument slit or imaging focal plane. It also means that replacing the existing acquisition

*sadkins@keck.hawaii.edu; Phone: (808) 885-7887; Fax: (808) 885-4464; http://www.keckobservatory.org/ 
cameras requires adapting the new cameras to existing optical and mechanical configurations, and makes it unlikely that a single standard configuration will be suitable for every instrument.

The existing acquisition cameras on the Keck I instruments are all small format ( $288 \times 384)$ frame transfer CCDs. The resulting small FOV is a significant contributor to observing overheads as the telescope operator identifies fields and locates guide stars. The acquisition cameras on the Keck II instruments have a larger FOV, but use full frame CCDs and require a shutter to avoid image smearing and excess background during readout. The priorities for the selection of the new acquisition camera hardware included improved sensitivity, fast split frame transfer to allow shutterless guiding, a compact package suited to the existing instrument envelopes, an industry standard interface for data and control, and a high level of maintainability.

Based on the concepts and priorities for the MAGIQ project we established a set of major objectives for hardware and software development activities. The major objectives for MAGIQ hardware development were an acquisition camera system consisting of a camera, mechanisms for focus adjustment and filter selection, and pickoff, collimation and focal reduction optics compatible with existing instrument configurations. The acquisition camera would be expected to provide a FOV allowing a $95 \%$ probability of finding a $\mathrm{V}=19$ guide star at the north galactic pole $(\mathrm{b}=90)$. The acquisition camera hardware was also expected to support specific capabilities including measurement of telescope image quality, characterization of observing conditions, and if possible, auxiliary photometric measurements.

The major objectives for the software were to provide all of the functionality of the existing acquisition and guiding software while improving routine tasks such as star list management, field identification, acquisition, and guiding. The software would also support the image quality monitoring functionality and provide closed loop control of telescope focus. The user interfaces would support improved workflow, improved communication between Observing Assistants (OAs) and observers, and diagnostic capabilities. A Keck Task Library (KTL) interface was also required to support scripting of acquisition and guiding operations, collection of image quality data in the headers of science data files, and procedures such as telescope pointing tests. The software also needed to support the interfaces required for communicating with new camera hardware and controlling mechanisms such as filter wheels, camera focus, etc.

\section{DEVELOPMENT PROCESS}

The design and development process for MAGIQ included three design phases each of which concluded with an external peer review. The first phase, system design ${ }^{5}$, was completed in December 2005 and resulted in a system requirements document covering each of the key design areas for MAGIQ, optics, mechanics, electronics and software. These requirements were flowed down from a set of user (observers and Observatory staff) driven high level requirements associated with the three major functional blocks of the MAGIQ system architecture: acquisition, guiding, and image quality monitoring.

The second design phase, preliminary design was completed in February 2006. During this phase the main activities included a detailed study of the optical and mechanical configurations required for the acquisition cameras in of each of the existing instruments, selection of the acquisition camera hardware, investigation of potential image quality monitoring techniques, and development of a comprehensive set of software design documents including software requirements and use cases. Software prototypes were developed for user interface validation and to support performance testing in areas such as image display rates and network bandwidth requirements. The optical and mechanical studies, along with the MAGIQ hardware requirements, led to the development of a concept for standard MAGIQ subassembly designs that could be implemented with a minimum of redesign in each of the acquisition camera upgrades. During the final design phase, detailed design, concepts for MAGIQ hardware components were developed into designs suitable for fabrication. Because of the anticipated development timescale, full scale development of the software was also started during this phase.

The first instrument to receive a MAGIQ upgrade was selected after consideration of both the potential benefits to observing efficiency, the reliability issues presented by each of the existing acquisition camera implementations, and the level of risk. The NIRSPEC ${ }^{6}$ instrument was ultimately selected as a lower risk choice that would provide an optimal platform for developing the full set of MAGIQ capabilities and components. It is relatively easy to access the annular FOV acquisition camera optical path and the instrument is equipped with a separate IR wavelength slit-viewing camera that would remain unchanged. This allowed demonstrating a dual channel configuration where guiding is done with the slit viewing camera while simultaneous image quality measurement is performed with the acquisition camera. 


\section{IMPLEMENTATION}

\subsection{Camera}

Since the detector is the heart of the camera, our first step in camera selection was to evaluate the specifications of commercially available CCD detectors with respect to relevant acquisition camera requirements, in particular guide star probability, centroid error, update rate, latency, pixel scale, and field of view. After considering a trade space encompassing these requirements it was apparent that a 1024 x 1024 pixel, thinned, backside illuminated CCD would be the most satisfactory choice. An additional consideration was the elimination of shutters in the acquisition cameras, a key source of reliability problems in the existing systems. This led directly to the requirement for a frame transfer CCD. With all of these considerations in mind we selected the E2V Technologies CCD47-20BT for the detector. A search was then made for cameras capable of supporting this detector and fitting within the mechanical space envelope available for acquisition cameras in our existing instruments. Six candidate cameras (four commercially manufactured and two open source designs) were evaluated with respect to seven decision criteria derived from the MAGIQ requirements: camera imaging performance (noise, stability, etc.), optical, mechanical, and electronic interfaces, operations effort (maintenance, repair, etc.), cost, and technical risk.

As a result of this trade study we concluded that the SciMeasure Analytical Systems Little Joe ${ }^{7}$ camera was the best choice for the standard MAGIQ acquisition camera. It is well matched to the CCD47-20, and delivers nearly detectorlimited performance, highlighted by low read noise and a high frame rate. The camera head is of small size, which simplifies the integration of this camera into existing instruments. Separate camera controller and power supply modules allow mounting flexibility, and the controller is implemented with three $3 \mathrm{U}$ Eurocard format circuit cards. These cards are readily accessible and interchangeable, greatly increasing the serviceability of the camera.

\subsection{Image Quality Monitoring}

The benefits to nighttime operations from image quality monitoring are better appreciated when the existing procedures for telescope focusing are considered. The Keck telescopes are focused by pistoning the secondary mirror. The secondary mirror is equipped with a drive system to adjust secondary piston and tip/tilt. The telescope control system (TCS) provides open loop correction for compensation of telescope focus as a function of temperature, elevation, rotator angle, and on Keck I to compensate for the action of the Cassegrain Atmospheric Dispersion Compensator (ADC). Rotator angle compensation is only implemented for the LRIS instrument. Two software programs, called Autofoc and $\mathrm{Mira}^{8}$, are used for telescope focusing. Autofoc pistons the telescope secondary through focus and the resulting image sizes are then evaluated to find the best focus. This technique does not provide any information on coma or other image aberrations. Mira uses the 36 images from the primary mirror segments that are produced when a known change in the shape of the primary mirror is introduced by the primary mirror active control system. Mira is capable of measuring focus, coma, and primary mirror segment stacking errors but requires that the instrument have an imaging mode. Both programs use direct imaging and they could be run on either the science instrument or the acquisition camera. However, the acquisition cameras in many of the instruments are not precisely parfocal with the science field, and some have problems with uniformity across the field. Because of these problems Mira is run only with science imagers. Autofoc is usually run with acquisition camera images, and at this point it is normally used only with HIRES or when the spectroscopic setup on another instrument makes changing to an imaging mode to run Mira undesirable. Autofoc typically takes 5 minutes to run. Each Mira run takes 5 to 10 minutes, depending on the instrument and various mode selection details for the analysis. Telescope focusing is done at the start of the night and then two to three additional times during the night, depending on the observing program. After large changes in elevation a change in telescope focus is often noted, also resulting in a Mira or Autofoc run.

The approach taken by MAGIQ to reduce the time lost to telescope focusing, and to support both continuous focusing and measurement of image quality is to provide a low order wavefront sensing capability. To guide the development of this capability the logs for both telescopes from Mira runs over a one year period (2004 to 2005) were analyzed. The results of this analysis are shown in Table 1 . The first column lists four sources of image quality degradation. The fourth source, segment figure errors, is provided for reference to scale the significance of the first three errors (segment figure errors are only measurable using the phasing camera system or PCS). The segment figure errors come from analysis of primary mirror segment figures made after segment exchanges on the Keck II telescope in 2003 and 2004. The second column gives values for secondary piston errors, secondary tip/tilt errors and stacking errors based on a study of the range of values reported by Mira prior to applying the required correction. The third column shows the impact on the full width half maximum (FWHM) of the Keck telescope image size from the various error contributors as determined from 
an optical model of the telescope. Secondary piston contributes mostly to wavefront focus while secondary tip/tilt contributes mostly to coma. The fourth column shows the current frequency with which each of the four sources of image quality degradation are measured and corrected and the fifth column shows the changes to the frequency of these corrections that results from implementation of MAGIQ control of the telescope secondary.

\begin{tabular}{|c|c|c|c|c|}
\hline Contributor & Image blur contribution & Impact & $\begin{array}{c}\text { Test/correction } \\
\text { frequency }\end{array}$ & $\begin{array}{c}\text { Test/correction } \\
\text { frequency - MAGIQ }\end{array}$ \\
\hline $\begin{array}{l}\text { Secondary } \\
\text { piston }\end{array}$ & $\begin{array}{c}0.25 " \text { to } 0.26 " \\
\text { (Mira or Autofoc) }\end{array}$ & $\begin{array}{l}13 \% \text { increase in FWHM for a } \\
0.5^{\prime \prime} \text { image }\end{array}$ & $\begin{array}{l}1 \text { to } 3 \text { per night plus } \\
\text { open loop correction }\end{array}$ & Continuous correction \\
\hline $\begin{array}{l}\text { Secondary } \\
\text { tip/tilt }\end{array}$ & $\begin{array}{l}0.11 " \text { to } 0.18 " \\
\text { (Mira only) }\end{array}$ & $\begin{array}{c}6 \% \text { increase in FWHM for a } \\
0.5^{\prime \prime} \text { image }\end{array}$ & 0 to 3 per night & Continuous correction \\
\hline Stacking & $\begin{array}{l}0.1 " \text { to } 0.2 " \\
\text { (Mira only) }\end{array}$ & $\begin{array}{c}8 \% \text { increase in FWHM for a } \\
0.5 " \text { image }\end{array}$ & $\begin{array}{l}0 \text { to } 3 \text { per night plus } \\
\text { open loop correction }\end{array}$ & Same \\
\hline Segment figure & $\begin{array}{l}0.16 " \text { to } 0.31 " \\
\text { (PCS only) }\end{array}$ & $\begin{array}{c}18 \% \text { increase in FWHM for a } \\
0.5 " \text { image }\end{array}$ & $\begin{array}{l}\text { Once every two years } \\
\text { (per segment) }\end{array}$ & Same \\
\hline
\end{tabular}

Table 1: Major contributors to Keck telescope image quality degradation

As indicated in Table 1 the largest errors found for secondary piston and tip/tilt would independently contribute $13 \%$ and $6 \%$ respectively to the FWHM of a 0.5 " image. Since Mira is known to reduce these errors to essentially zero at the time it is run, the log data implies that errors of these magnitudes routinely build up during the observing night. This also suggests that the open loop correction provided by the TCS is not capable of fully correcting secondary piston errors. In addition, no open loop correction is provided for secondary tip/tilt although it is known that some secondary tip/tilt error is introduced by telescope elevation changes. The amount of image degradation that should be tolerated due to focus and tip/tilt errors is a matter of some judgment but we clearly should expect to make an improvement that is significant when compared to the levels shown in Table 1. Besides the effect on image quality, minimizing tip/tilt errors is essential for MAGIQ because of the curved focal surface of the Keck telescope design. To first order the center of curvature of this surface is determined by the collimation of the secondary mirror. If we expect to use a camera located at a position that is offset from the optical axis of the telescope, a condition that is true for all of the acquisition cameras, we need the displacement of the focal surface due to the curvature to remain constant at all rotator angles. This requires accurate collimation of the secondary mirror. After reviewing the reported performance of Mira secondary focus and tip/tilt control, we determined that a requirement of a $2 \%$ maximum increase in image FWHM (due to secondary piston or tip/tilt combined in quadrature with a seeing disk of $0.5 "$ ) was consistent with the measured performance of Mira.

As noted earlier, the acquisition camera architecture at WMKO is based on providing dedicated cameras for each instrument. Adding image quality monitoring within the constraints of the limited space available in the instruments and for a variety of optical designs is a significant challenge. After reviewing a number of options we concluded that a method is needed for low order wavefront sensing that can be implemented as an integral part of the new MAGIQ acquisition cameras. This approach also imposes the additional constraint that it must be possible to use the MAGIQ camera for both guiding and wavefront sensing.

After reviewing the configurations of the instruments we selected two methods as candidates for integral wavefront sensing, the Shack-Hartmann (SH) technique or a technique based on a single defocused image, called "donut" by the authors of a paper ${ }^{9}$ describing an algorithm for determining low order aberrations from a defocused image. The $\mathrm{SH}$ pattern could be created by a prismatic optical element located at a pupil plane in the optical path of the acquisition camera. Placing a weak defocusing lens in this same location could create the defocused image for the donut method. A mechanism could then be used to move either wavefront sensing optical element in and out of the optical path of the acquisition camera. The ability to remove the wavefront sensing element means that the full field of the camera is useable for acquisition. In particular, since MAGIQ is intended to improve the Keck telescope acquisition process (made more difficult because of limited blind pointing accuracy) by providing a larger FOV, it is necessary to provide for a mode where the SH prism is out of the beam. It also allows the operator to choose a "guiding only" mode for fields where only a very faint guide star is available. The deployment mechanism also provides the ability to interleave wavefront sensing frames with guiding frames.

The donut method has several unique features that make it suitable for application to MAGIQ. Like curvature sensing the donut method uses defocused images for wavefront sensing, but the method requires only one image and the amount of defocus needed is typically much smaller. Using defocused images is also attractive in astronomical applications 
because it can be done on any science detector with a focus adjustment independent of the telescope. During the MAGIQ preliminary and detailed design phases we conducted on sky tests of the donut method but we were unable to validate its performance in the limited engineering time available. After simulations of the expected performance of a low order SH method we selected this method as the baseline wavefront sensing technique for MAGIQ.

Three different SH prism patterns were considered as illustrated in Figure 1, providing 4, 5 or 6 subapertures over the telescope pupil. To evaluate the performance of the SH method using these subaperture configurations a modal wavefront estimation technique was used ${ }^{10,11}$. The performance of a modal wavefront estimator was evaluated for each of the subaperture configurations. Zernike coefficients up to Z7 and Z8 (coma) were estimated; with the gradient of each Zernike approximated by a finite difference method over a grid in the telescope pupil and averaged over the appropriate geometry for 4, 5 and 6 subapertures. A matrix was compiled from the appropriate gradients and the inverse of this matrix was determined using the singular value decomposition routine in IDL. In all cases the matrix inversion was well behaved. The error propagator was calculated as well. The values of the reconstructor were checked for correctness by reconstructing pure tilt wavefronts.

Figure 1: Subaperture configurations, 4 subapertures (left), 5 (center), and 6 subapertures (right). The hexagonal Keck telescope pupil is indicated by the blue polygon.
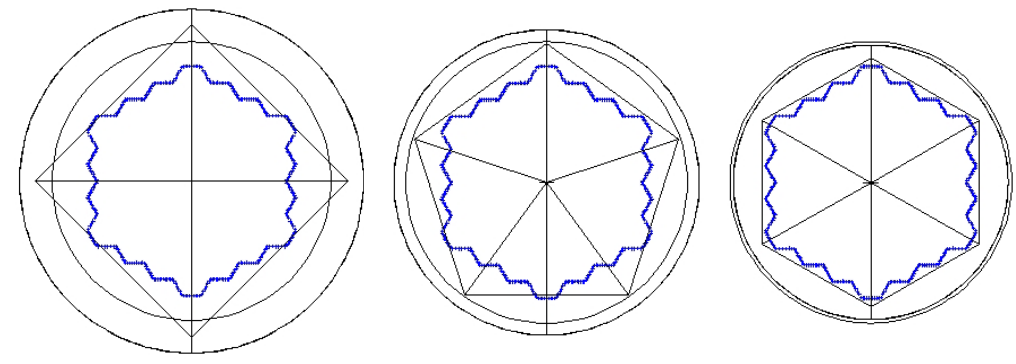

In order to convert from Zernike aberrations to collimation moves of the Keck secondary a Zemax model of the Keck telescope was used to estimate the Zernike amplitude when the secondary was defocused and tilted. The error propagators for Zernike focus and coma were then applied to centroid error estimates from noise based SH image simulations and converted to secondary positions using the conversion factors estimated from the telescope model. The results are compared to the MAGIQ requirements for secondary piston and tip/tilt in Table 2.

\begin{tabular}{|l|c|c|c|c|c|}
\hline & \multirow{2}{*}{$\begin{array}{c}\text { MAGIQ req. for } \\
\text { Image blur contributor }\end{array}$} & \multicolumn{3}{|c|}{$\begin{array}{c}\text { Performance vs. number of } \\
\text { subapertures }\end{array}$} \\
\hline Secondary piston error, mm & $\mathbf{0 . 5}$ FWHM & $\begin{array}{c}\text { Number of } \\
\text { measurements }\end{array}$ & $\mathbf{4}$ & $\mathbf{5}$ & $\mathbf{6}$ \\
\cline { 3 - 6 } & \pm 0.030 & 1 & 0.014 & 0.013 & 0.012 \\
\cline { 3 - 6 } & & 30 & 0.003 & 0.002 & 0.002 \\
\hline $\begin{array}{l}\text { Secondary tip/tilt error, arc } \\
\text { seconds }\end{array}$ & \pm 40 & 1 & 59 & 40 & 33 \\
\cline { 3 - 6 }
\end{tabular}

Table 2: SH measurement error estimates

In this application, it is necessary to reconstruct Zernike modes up to Z7 and Z8 (coma). Conventionally this would require at least 9 subapertures for SH wavefront sensing. However, since MAGIQ must use comparatively long time scale measurements in order to discount atmospheric effects, it is practical to accumulate a number of wavefront measurements and use co-adding or averaging to reduce the measurement error. The value shown for 30 measurements assumes that averaging the measurements yields an improvement of $\sqrt{3} 0$. This analysis suggested that any of the SH configurations were capable of providing estimates of secondary piston and tip/tilt that would meet our performance requirements. Based on the improved secondary tip/tilt measurement performance we selected a 5 subaperture configuration for the NIRSPEC hardware upgrade.

SH wavefront sensing requires calibration to establish the reference or "null" position, in this case corresponding to zero focus and coma. This calibration will be established every night by performing a Mira run at the start of the night. Immediately after the Mira run a MAGIQ acquisition camera auto focus sequence will be run to ensure that the acquisition camera is parfocal with the science instrument.

Calibration will be required to verify the SH spot displacement as a function of secondary piston and tip/tilt and will also be required to compensate for field dependent aberrations in the camera FOV, such as those caused by locating the camera off axis. This calibration may also need to be performed at different rotator angles, and problems such as 
differential flexure affecting focus between the science field and the acquisition camera must be considered and addressed.

For the SH method the location of the guide star must not be too close to the edge of the FOV, ensuring that when the SH prism is placed in the beam no part of the SH pattern falls outside of the FOV. The SH method is unlikely to be suitable for use with a slit viewing camera, particularly since these cameras typically have a small FOV, reducing the probability of finding a suitable star for wavefront sensing, and also because the image reflected from the slit plane contains additional aberrations. Fortunately, for WMKO instruments when a slit viewing camera is provided it is normally accompanied by imaging capability for acquisition, either with a second camera (LRIS, NIRSPEC) or a split field (DEIMOS, ESI). The only exception is HIRES, but its small slit camera FOV makes it likely that a second imaging camera will need to be implemented for acquisition in order to get the required FOV for MAGIQ, and this will also permit implementation of SH wavefront sensing. It should also be noted that for configurations that provide separate slit viewing and imaging cameras, when guiding with the slit viewing camera, the imaging camera can be used for wavefront sensing at the same time.

\subsection{Acquisition Camera Opto-mechanical Design}

The detailed design for the MAGIQ acquisition camera has resulted in a set of standard subassemblies. With limited customization, normally confined to the mounting details of the subassembly, these components can be used in each acquisition camera upgrade, reducing the overall design effort for each upgrade. This is an important benefit since each upgrade must be packaged differently and will require custom pickoff and collimating optics, still a significant expense. There are four standard subassemblies, the acquisition camera head, the focus adjustment mechanism, the filter wheels and the wavefront sensing mechanism.

The MAGIQ acquisition camera head is a modified version of the SciMeasure Little Joe camera head and is shown in Figure 2. The focal reducing lens is mounted on a short barrel, equipped with a standard Nikon F series bayonet mount, which can be adjusted for centration and tip/tilt. These adjustment features were added to permit precise alignment of the focal reducing lens with the image plane of the CCD. The location of this image plane with respect to the CCD package mounting plane is not tightly specified due to the process used to mount the CCD in the package. Because the acquisition camera will be used as part of a wavefront sensing system, as well as for imaging, proper alignment of the focal reducer lens to the CCD image plane is essential. A bench alignment procedure is used to align each focal reducer with the CCD. Due to the highly repeatable nature of the Nikon F mount and the quality of the focal reducing lens the resulting alignment is maintained when the focal reducing lens is removed and replaced.

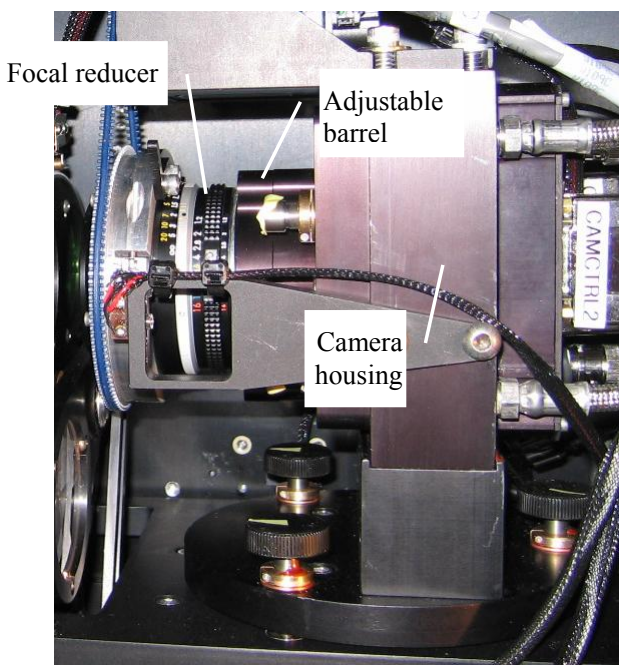

Figure 2: MAGIQ Acquisition Camera Head

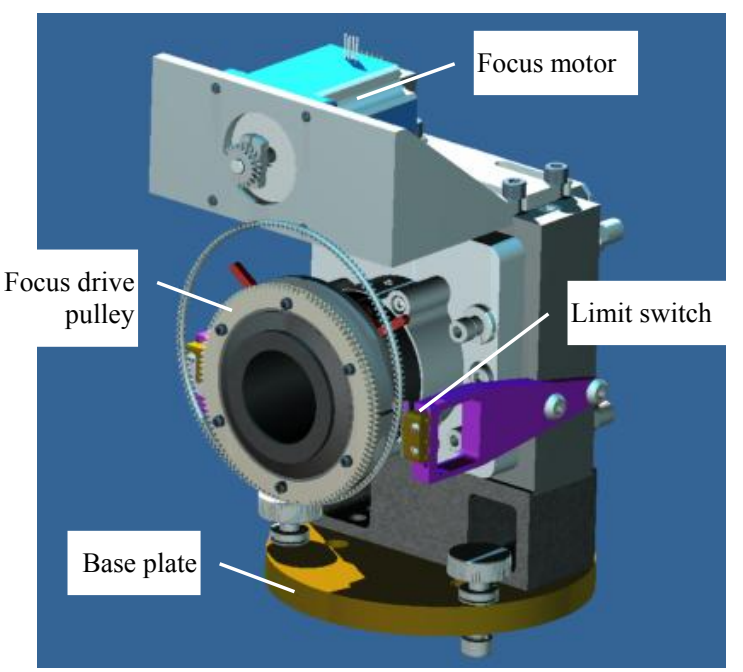

Figure 3: MAGIQ Acquisition Camera Assembly

A base plate, shown in Figure 3, is kinematically mounted to a support plate in each instrument and permits alignment of the camera head. When this is combined with the precise alignment of the focal reducer to the CCD this allows interchanging cameras for service with a minimal effect on alignment of the optical path. Also shown in figure 3 is the 
focus adjustment mechanism. This mechanism uses a large pulley clamped to the focal reducer focus ring and driven via a timing belt by a NEMA size 23 stepper motor equipped with a shaft encoder The range of focus is controlled with mechanical forward and reverse limit switches nominally set to allow $83^{\circ}$ of rotation. To protect the focal reducer lens in the event of a problem with the motor control, hard stops are provided, limiting rotation to $100^{\circ}$. Figure 3 shows the timing belt installed on the large pulley only in order to simplify the CAD model.

The MAGIQ filter wheel and wavefront sensing deployment mechanisms are shown in Figure 4. Each filter wheel can hold five filters with one open position. The filter wheels are edge driven via an anti-backlash pinion gear by NEMA size 23 stepper motors equipped with shaft encoders. A mechanical switch is provided as a home position switch. Driving the stepper motor a full step provides an acceptable resolution of $2.8^{\prime}$, or $0.22 \mathrm{~mm}$ at the edge of a filter. This allows powering off the stepper motor and using the cogging torque to hold the filter wheel in the proper position, limiting power consumption. Two filter wheels are normally used, one for photometric band pass filters, and one for neutral density filters.

MAGIQ supports two different operating modes for wavefront sensing, "on demand" and "continuous". The on demand mode causes the wavefront sensing element to be inserted into the acquisition camera beam, where it remains for the required number of frames (typically 30) and is then removed. The continuous mode causes the wavefront sensing element to be inserted into the beam for 1 of every $\mathrm{n}^{\text {th }}$ (user determined) frames, this mode is used during guiding to allow simultaneous wavefront sensing and guiding. While it is in principle possible to guide using the SH pattern, the additional complications that this would create are avoided by interleaving wavefront sensing frames (element in beam) with guiding frames (element out of beam).

A 5 faceted prism with a facet angle of $1^{\circ}$ with respect to a plane normal to the optical axis (producing a pattern with $\sim 5^{\prime \prime}$ radius) is used as the wavefront sensing element. This element is inserted into the beam by the mechanism shown in Figure 4. A NEMA 34 frame size dc servomotor with a peak torque rating of $23.6 \mathrm{lb}$-inch equipped with a shaft encoder is used to actuate an eccentric drive that rotates in one direction. With every revolution of the motor the optical element rotates $90^{\circ}$ into the beam, reverses direction, and rotates $90^{\circ}$ out of the beam. The optical element holder is connected by an aluminum linkage to the motor shaft and the encoder index signal is used as an absolute position reference. This comparatively robust motor is used to permit moving the wavefront sensing element in and out of the beam in $300 \mathrm{~ms}$, the minimum exposure time for the MAGIQ camera. This allows interleaved guiding and wavefront sensing frames with a 3 frame gap in the guide frame stream for every wavefront sensing frame (one frame to move the element in, one wavefront sensing frame, one frame to move the element out) at the maximum frame rate. Because the mechanism is expected to have a relatively high duty cycle ( $\sim 1000$ cycles per night if continuous mode is routinely used) the design uses all rotary motion with high quality bearings. An operational lifetime of $>10$ years is expected based on MTBF data for the components used.

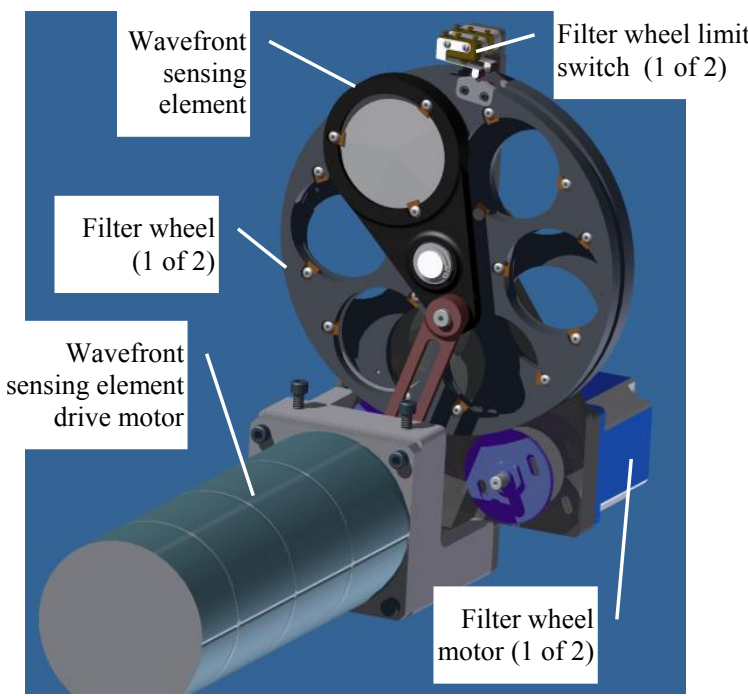

Figure 4: MAGIQ filter wheels and wavefront sensing mechanism

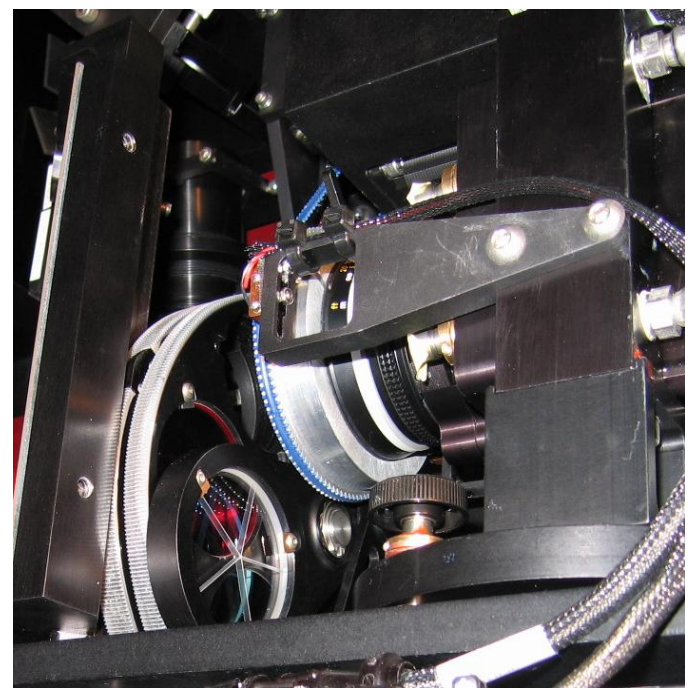

Figure 5: Wavefront sensing element 
In Figure 5 a portion of the two filter wheels can be seen at the left side of the image, with the 5 faceted prism in the wavefront sensing element holder just to the right of the filter wheels at the bottom left in the image. Above and to the right, near the center of the image, the focal reducer focus drive pulley can be seen mounted on the focus ring of the focal reducing lens. One of the focus mechanism limit switches can also be seen near the center of the image on a bracket attached to the camera housing.

\subsection{NIRSPEC Configuration}

The NIRSPEC acquisition camera is mounted on the front of the NIRSPEC instrument in an enclosure that houses a calibration unit and the acquisition camera. The front of NIRSPEC is shown in Figure 6 with the calibration unit cover removed. The calibration unit is at the upper left with the acquisition camera optical components, mechanisms (filter wheel, wavefront sensing element, camera focus) and camera head below. Figure 7 shows the new MAGIQ acquisition camera as installed, with the guider pickoff mirror at the left side, followed by the filter wheels, wavefront sensing element, focal reducer, and camera head.

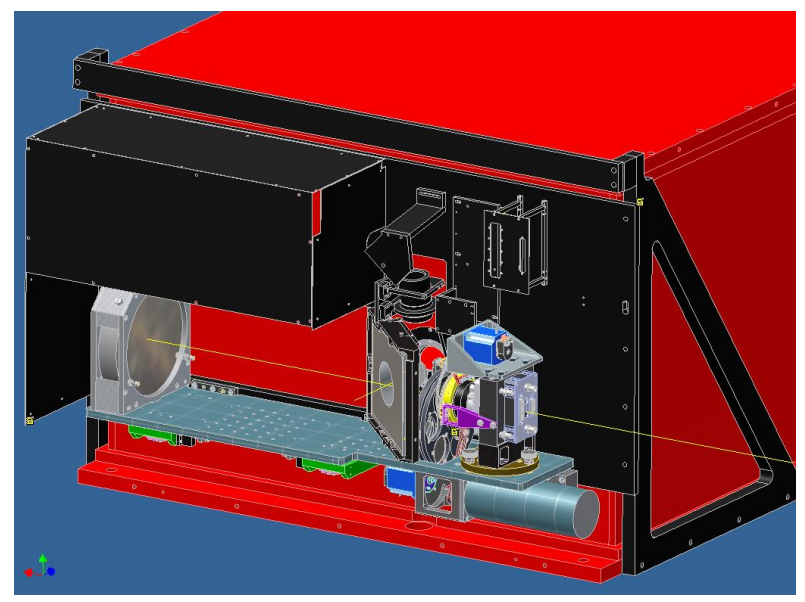

Figure 6: Front of the NIRSPEC instrument showing the calibration unit and the upgraded acquisition camera hardware

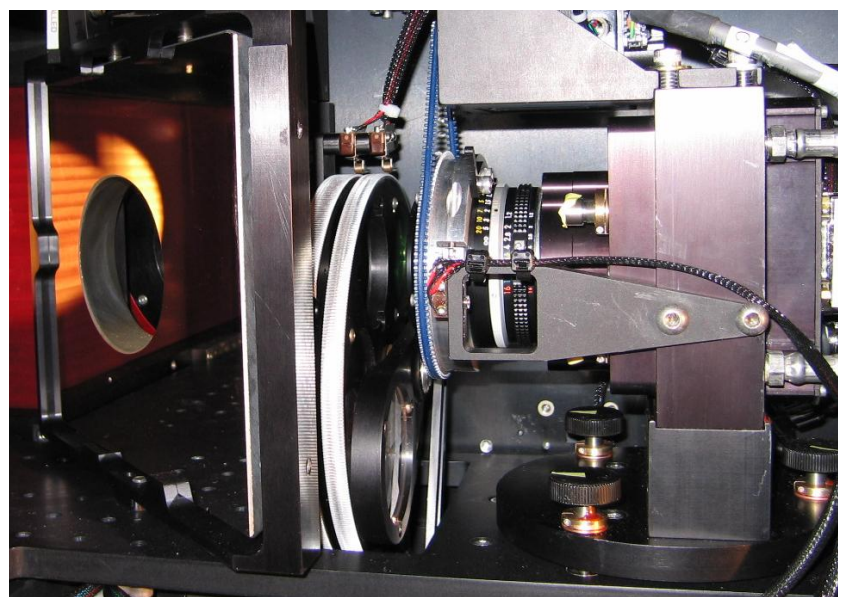

Figure 7: NIRSPEC/MAGIQ Acquisition Camera

The optical configuration of the NIRSPEC/MAGIQ acquisition camera is shown in Figure 8. This optical configuration is similar to the original NIRSPEC acquisition camera, but the pick-off mirror and collimator have protected silver coatings for improved throughput. The pickoff mirror mount has an improved design to permit a more precise alignment of the guider optics to ensure uniform focus over the whole guider field.

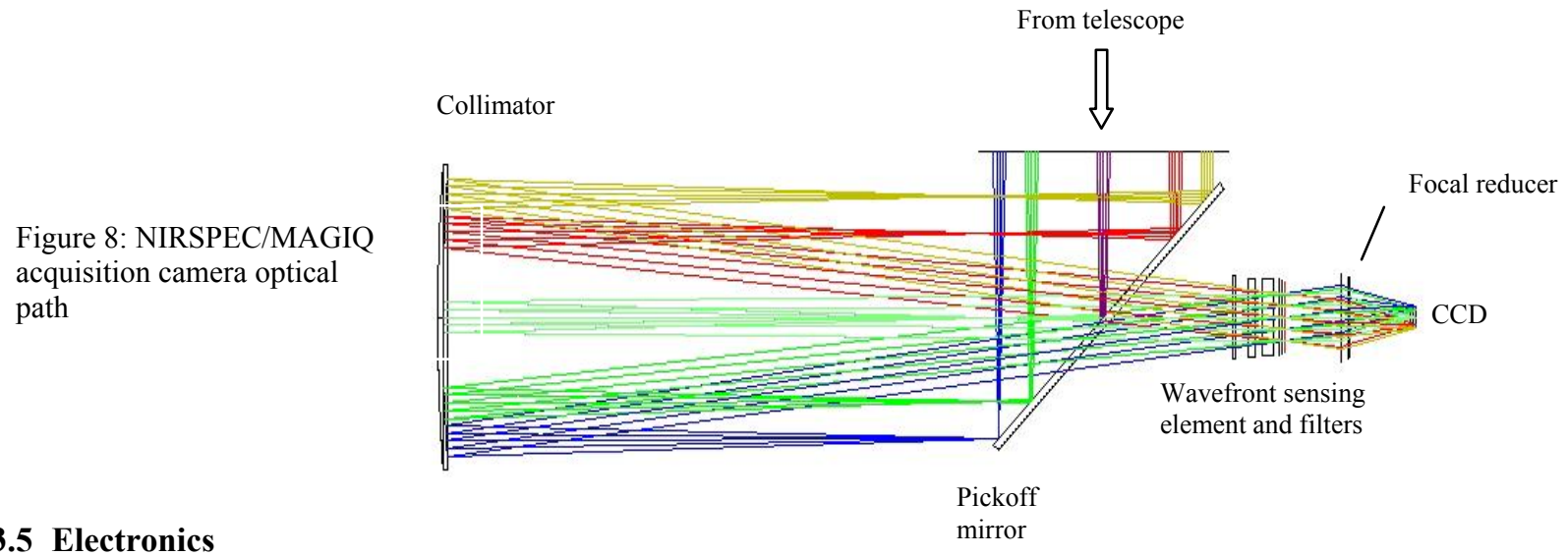

The acquisition camera electronics consists of the SciMeasure camera controller, a Galil DMC-4060 6 axis stand-alone motion controller and power supply for the focus, filter wheel and wavefront sensing drives, a power controller, and a network switch. The network switch is connected through an optical fiber to Ethernet interface to a fiber link to the MAGIQ camera server computer. The motion controller and the power controller communicate via this fiber in a private 
network with the MAGIQ camera server. The motion controller also provides digital inputs, conditioned by pulse stretching circuits, to sense the camera frame timing signals. These signals are used in conjunction with a motion controller digital output signal and a motion controller subroutine to time the MAGIQ camera exposures. This approach simplifies synchronization of the wavefront sensing element motions in the continuous wavefront sensing mode. The SciMeasure camera controller supports a Camera Link interface, and this interface communicates via optical fiber with a frame grabber card located in the MAGIQ camera server. Both camera control I/O and image data flow over the Camera Link interface. For the NIRSPEC/MAGIQ implementation the acquisition camera electronics are enclosed in a new glycol cooled NEMA 4 enclosure equipped with internal EIA equipment mounting rails. This was done to reduce the power dissipation in the NIRSPEC electronics enclosure and to help in avoiding electromagnetic interference between the new electronics and the NIRSPEC electronics.

\subsection{Software}

The software is the second major component of MAGIQ and its overall architecture can be described by a hybrid three tier model with a presentation tier (user interface and keyword interface), an application tier (acquisition, guiding, image quality monitoring, and subsystem interfaces), and a data tier (subsystems including telescope control, camera control, star catalogs, etc.). The software provides improved user interfaces for both the observing assistants (telescope operators) and the observers. It provides a number of workflow improvements as well as the functionality necessary to support the new acquisition camera and the image quality monitoring functionality.

During the preliminary design phase of MAGIQ it was realized that supporting the existing guide cameras would provide a number of benefits including overall risk reduction, early availability of the improvements to acquisition, guiding, and work flow, and additional operating efficiency benefits. As a consequence, the acquisition and guiding functionality of the MAGIQ software was tested and released for initial use prior to upgrading the NIRSPEC acquisition camera. This was important for operations because prior to the hardware upgrade it was possible to revert to the older acquisition and guiding software (xguide) if a problem with the MAGIQ software were found that would cause a loss of observing time. Once the hardware was upgraded, only the MAGIQ software could be used.

Once the hardware upgrade was installed the process of commissioning was greatly eased by the level of testing already completed for the software. After verifying and adjusting the coordinate space for the new camera, it was immediately possible to begin guiding, allowing the various hardware performance tests to proceed smoothly. A more extensive treatment of the software design and functionality is given elsewhere in these proceedings ${ }^{12}$.

\section{PERFORMANCE}

Prior to installation in NIRSPEC, the upgrade hardware was integrated, aligned and tested as a unit in the laboratory. The laboratory was equipped with a complete optical test bench including an integrating sphere and highly stable light source for photon transfer measurements and other camera/detector characterizations, and a point source with telescope simulator optics to evaluate the overall alignment and image quality of the new acquisition camera optics. This also allowed initial verification of the 5 faceted prism used as the wavefront sensing element. An alignment procedure was fully developed during the detailed design phase, and this included the manufacture of a number of jigs and fixtures to be used in laboratory alignment and during installation on the NIRSPEC instrument at the Observatory's summit facilities.

Laboratory testing of the upgrade included operation with the MAGIQ software to confirm the basic performance of the camera interface and related software. The optical test bench will continue to be an important tool for the development of future upgrades, as well as providing a facility for the testing and initial alignment of the camera, an important part of the interchangeability required to support rapid response in the event of a hardware failure. The performance of each new camera is verified using a comprehensive incoming test procedure developed as part of the MAGIQ project.

On sky testing of the NIRSPEC/MAGIQ hardware upgrade included verification of the basic optical performance (image formation, focus uniformity, vignetting, internal reflections and ghosting), testing of all of the mechanisms (filter wheels, focus mechanism, wavefront sensing element deployment), measurement of sensitivity, and calibration of the wavefront sensing mode. Table 4 summarizes the key performance specifications for the MAGIQ acquisition camera hardware along with the actual performance measured for the appropriate parameters during on sky testing. The actual values reported in Table 4 are for the camera installed in NIRSPEC, and represent just two of the numerous performance verifications performed in the camera testing. 
The parameters given under guide star probability (pixel scale, FOV, sensitivity) represent derived parameters in support of the required probability for finding suitable guide stars. The sensitivity estimates listed as specified values are the predicted values based on MAGIQ sensitivity calculations, taking into account the detector manufacturer's specifications for $\mathrm{QE}$, and measured values for read noise and dark current, along with the specified values for telescope and acquisition camera optics transmission.

\begin{tabular}{|c|c|c|c|c|}
\hline Parameters & $\begin{array}{l}\text { Specification } \\
\end{array}$ & Actual Performance & Units & Notes \\
\hline Camera & SciMeasure Little Joe & $\checkmark$ & - & \\
\hline Detector & $\begin{array}{c}\text { E2V Technologies CCD47- } \\
\text { 20BT }\end{array}$ & $\checkmark$ & - & \\
\hline $\mathrm{QE}$ (V band) & 0.85 & Not measured & - & \\
\hline Size & $1024 \times 1024$ & $\checkmark$ & pixels & \\
\hline Read noise & 10 & 9.7 & $\mathrm{e}^{-}$ & 1 \\
\hline Frame rate & $\geq 1$ & 3.5 (maximum) & full frames/s & \\
\hline \multicolumn{5}{|l|}{ Optical transmission } \\
\hline Telescope & 0.59 & - & - & 2 \\
\hline Acquisition camera & 0.6 & 0.78 & - & \\
\hline Guide star probability & $>95 \%$ & - & - & 3 \\
\hline Pixel scale & 0.21 & 0.2 & arc seconds/pixel & \\
\hline FOV & 12.85 & 9.8 & square arc minutes & 4 \\
\hline \multicolumn{5}{|l|}{ Sensitivity } \\
\hline $\mathrm{V}$ band & $\mathrm{V}=20.8$ for $\mathrm{SNR}>10$ & $\mathrm{~V}=20.95$ for $\mathrm{SNR}>10$ & - & 5 \\
\hline $\mathrm{R}$ band & $\mathrm{R}=20.5$ for $\mathrm{SNR}>10$ & $\mathrm{R}=20.65$ for $\mathrm{SNR}>10$ & - & \\
\hline \multicolumn{5}{|l|}{ Wavefront sensing } \\
\hline $\begin{array}{l}\text { Secondary mirror } \\
\text { focus error }\end{array}$ & \pm 0.030 & \pm 0.009 & $\mathrm{~mm}$ & 6 \\
\hline $\begin{array}{l}\text { Secondary mirror } \\
\text { tip/tip tilt error }\end{array}$ & \pm 40 & $< \pm 20$ & arc seconds & 7 \\
\hline
\end{tabular}

\section{Notes:}

1. At the maximum frame rate

2. Nominal value for 3 surfaces, 0.84 /surface minimum from 0.32 to $1 \mu \mathrm{m}$

3. At the galactic pole for $\mathrm{V}=19$

4. 1.85 square arc minutes lost due to central hole for science field

5. 60 degrees from the full moon, sky brightness of $\mathrm{V}=18.654$

6. Telescope secondary piston measurement error, actual values are standard error for piston vs. Z4

7. Telescope secondary tip/tilt measurement error, actual values are standard error for piston vs. Z7, Z8

Table 3: NIRSPEC/MAGIQ Acquisition Camera Specifications and Actual Performance

Figure 9: Measured and predicted sensitivity

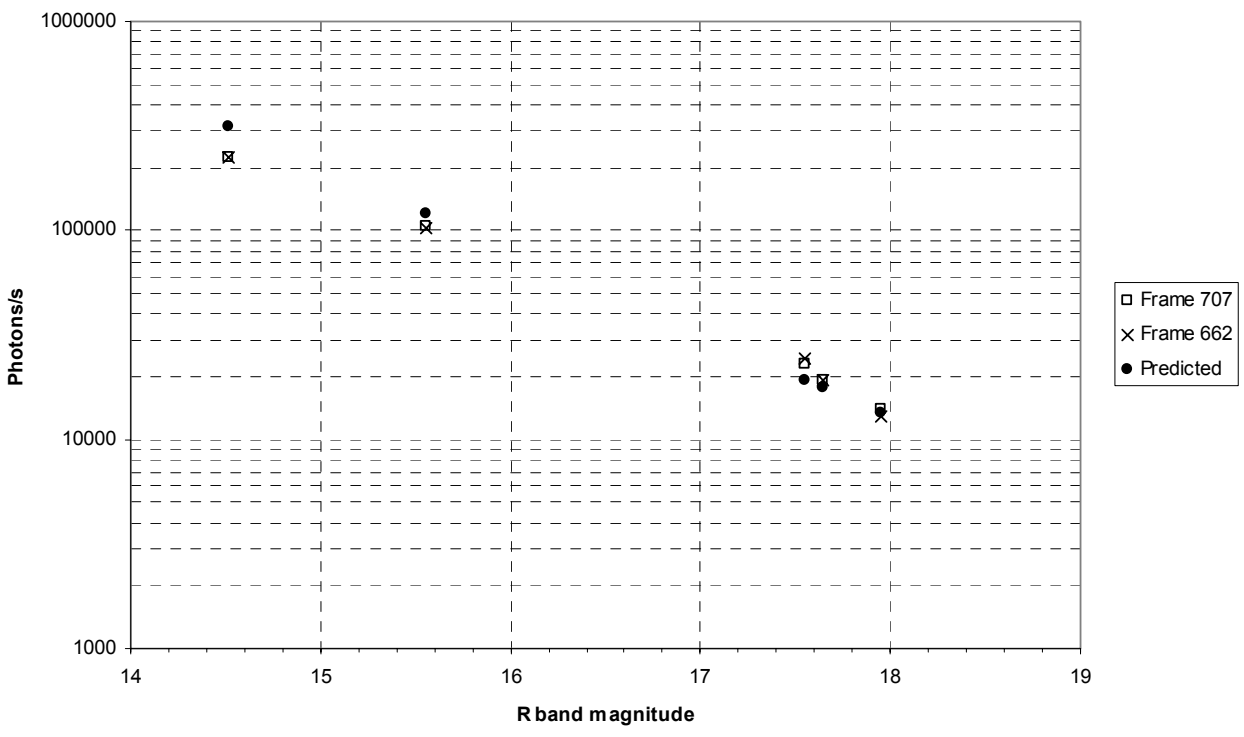


Sensitivity and photometric performance was initially evaluated using images of the globular cluster M92 (NGC 6341) taken in all three of the NIRSPEC/MAGIQ photometric bands (V, R, and I) to measure the sensitivity of the acquisition camera. The results for 5 stars in $\mathrm{R}$ band are shown in Figure 9 along with the predicted sensitivity. As of the time of writing this paper (mid-2008) the NIRSPEC/MAGIQ acquisition camera is fully commissioned for routine operations in acquisition and guiding. Initial testing of the sensitivity has been completed, but additional observations are needed to establish the stability and calibration for photometry.

Commissioning of the wavefront sensing capability is currently underway in a three step process. The first step, initial determination of the focus and tip/tilt measurement response has been completed. The left side of Figure 10 shows the values obtained for Zernike focus (Z4) as a function of induced secondary piston. There are two data sets shown along with the best straight line fit to each set of data. The data was generated by first acquiring a suitable star and performing a Mira to correct the telescope focus and tip/tilt and then systematically pistoning the secondary in $50 \mu \mathrm{m}$ increments about best focus and acquiring a total of 30 seconds of wavefront sensing images at each position with the SH prism in the beam. The fit is slightly poorer for the $59^{\circ}$ data due to the fact that during the time period required for the measurements the greater change in elevation resulted in additional secondary mirror motion during the test. The quality of the straight line fits, and the low standard error $(9 \mu \mathrm{m})$ is encouraging and allows an initial calibration of the piston measurement for use in further testing.

The right hand side of figure 10 shows the values of $Z 7$ and Z8 (coma) as a function of induced secondary tilt in $\mathrm{x}$. The same procedure as that used for the focus data set was followed, and after a Mira correction was applied the secondary was systematically tilted in 20 " increments about the zero coma position. The changes in both Z7 and Z8 are due to the axis of secondary tilt not being exactly aligned with acquisition camera coordinate system. Similar results were obtained for the y-axis. The straight line fit is not quite as good, but the standard error of the fit at $<20$ " for both $\mathrm{x}$ and $\mathrm{y}$ is within the MAGIQ requirements and allows an initial calibration of the tip/tilt measurement for use in further testing.
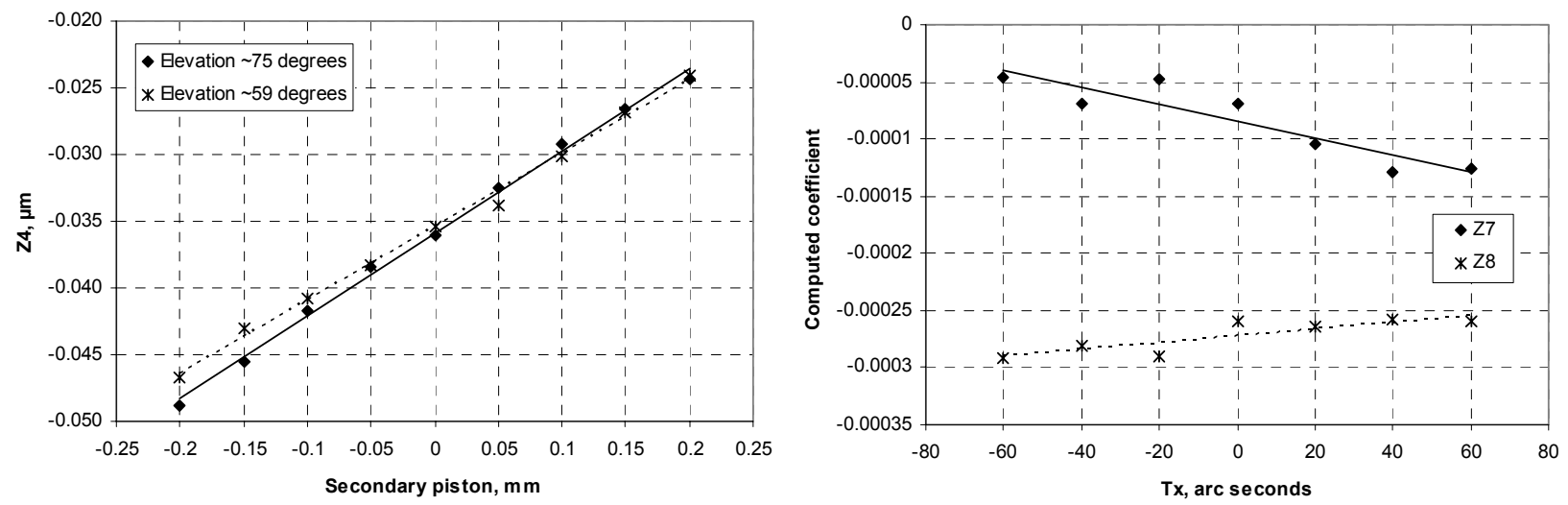

Figure 10: Initial calibration of wavefront sensing measurement, Z4 vs. induced piston (left graph), and Z7 and Z8 vs. induced $\mathrm{x}$-axis tip/tilt (right graph), similar results were obtained for $\mathrm{y}$-axis tip/tilt

The MAGIQ image quality monitoring user interface is used to take the wavefront measurements, providing continuous graphical display of Zernike terms derived from the measurements up to Z8, as well as providing displays of image FWHM and atmospheric transparency. As discussed earlier, MAGIQ offers two operating modes for wavefront sensing, on demand and continuous, and both can be used for measurement only or to correct the position of the telescope secondary. The continuous mode measurements can be applied at regular, user selected intervals. The interval between applications of continuous mode measurements is determined by the rate at which secondary piston and tip/tilt errors build up, a function of the rate of change in elevation, and typically between 5 and 7 minutes.

The next steps in the commissioning of the wavefront sensing capability will take place in July 2008. A series of tests will use MAGIQ wavefront sensing to perform secondary mirror position measurements and apply the corrections; the results of each test will then be verified using the Mira software and the calibration refined. Once these tests are successfully completed MAGIQ wavefront sensing will be released for measurement only operation during regular NIRSPEC observing. In this period, each time a Mira run is called for, MAGIQ will take wavefront sensing measurements before and after the Mira run. The results will be logged and then evaluated to confirm the reliability of 
the measurements. The final step will be to release wavefront sensing for full control of the telescope secondary in both on demand and continuous mode.

\section{CONCLUSIONS}

The MAGIQ acquisition camera is a set of standard subassemblies that can be used in each acquisition camera upgrade reducing the overall design effort for each upgrade. The first instrument (NIRSPEC) has received a hardware upgrade that was commissioned in March 2008. The MAGIQ software has been released for acquisition and guiding for NIRSPEC and we are in the process of testing and calibrating the wavefront sensing functionality with NIRSPEC. The next hardware upgrade planned is for the LRIS instrument, and we are planning to complete commissioning of the MAGIQ software for the legacy cameras on the LRIS, HIRES and DEIMOS instruments in the near future.

\section{ACKNOWLEDGEMENTS}

This material is based in part upon work supported by the National Aeronautics and Space Administration under Grant No. NNG05GC95G issued through the NASA Astronomy and Astrophysics Research and Analysis program. The W. M. Keck Observatory is operated as a scientific partnership among the California Institute of Technology, the University of California, and the National Aeronautics and Space Administration. The Observatory was made possible by the generous financial support of the W. M. Keck Foundation.

\section{REFERENCES}

[1] Boyer, C., Sebag, J., Rigaut, F. J., Chan, S., Carter, C. J., "Gemini Wavefront Sensing Subsystems", Proc. SPIE, Volume 4837, pp. 502-513, 2003.

[2] Cullum, M. J., Franza, F., Brunetto, E., "Sensing systems for active control of the VLT", Proc. SPIE Vol. 2871, pp. 99-104, 1997.

[3] Usuda, T., Takato, N., Morino, J., Kosugi, G., Miyashita, A., Noumaru, J., Kanzawa, T., Hayashi, S. S., Letawsky, M., Yamashita, T., Itoh, N., Tanaka, W., "Subaru Telescope: current performances and future upgrade plans", Proc. SPIE, Volume 4837, pp. 831-842, 2003.

[4] Schechter, P. L., Burley, G. S., Hull, C. L., Johns, M., Martin, H. M., Schaller, S., Shectman, S. A., West, S. C., "Active Optics on the Baade 6.5-m (Magellan I) Telescope", Proc. SPIE, Vol. 4837, pp. 619-627, 2003.

[5] Adkins, S. M. and R. H. Matsuda, "A new acquisition, guiding and image quality monitoring system for the W. M. Keck Observatory”, Proc. SPIE, Volume 6269, 2006.

[6] McLean, I. S., Becklin, E. E., Bendiksen, O., Brims, G., Canfield, J., Figer, D. F., Graham, J. R., Hare, J., Lacayanga, F., Larkin, J. E., Larson, S. B., Levenson, N., Magnone, N., Teplitz, H., Wong, W., "Design and development of NIRSPEC: a near-infrared echelle spectrograph for the Keck II telescope", Proc. SPIE Vol. 3354, p. 566-578, 1998.

[7] Duvarney, R., Bleau, C., Motter, G., Dekany, R., Troy, M., Brack, G., "SciMeasure wavefront sensor cameras and their application in the Palomar adaptive optics system", Optical Detectors for Astronomy II: State-of-the-Art at the Turn of the Millenium, 4th ESO CCD Workshop, 1999.

${ }^{[8]}$ Kwok, S. H. and A. Conrad, "Mira: a case study", Proc. SPIE Vol. 4848, pages 414-424, 2002.

[9] Tokovinin, A. and S. Heathcote, "Donut: Measuring Optical Aberrations from a Single Extra-focal Image", PASP, 118, 1165-1175, 2006.

[10] Cubalchini, R., "Modal wave-front estimation from phase derivative measurements", J. Opt. Soc. Am., Vol. 69, No. 7, 972-977, 1979.

[11] Gendron, E. and P. Lena, "Astronomical adaptive optics I. Modal control optimization", Astronomy and Astrophysics, 291, 337-347, 1994.

[12] Kwok, S. H., Johnson, J., Adkins, S. M., McCann, K., "The Software for MAGIQ: a new acquisition, guiding and image quality monitoring system at the W. M. Keck Observatory", Proc. SPIE 7019-10, June 2008. 